\title{
THE SIMULATION OF NON-ISOLATED EXPANDING GRAVITATIONAL SYSTEMS
}

\author{
G. JANIN \\ European Space Operations Centre, Darmstadt, F.R.G. \\ and \\ M. J. HAGGERTY \\ Centre for Statistical Mechanics and Thermodynamics, \\ The University of Texas at Austin, Tex., U.S.A.
}

\begin{abstract}
Numerical experiments on the gravitational $N$-body problem are reviewed for the case of nonisolated systems. The effect of an external field on expanding cubical systems is discussed.
\end{abstract}

\section{Numerical Experiments on Non-Isolated Spherical Systems}

Very few cases of non-isolated gravitational systems have been studied by the method of numerical experiments. Usually the gravitational system is defined in a universe where everything else is excluded. This is, of course, a non-realistic approximation.

Among the attempts to take into account an external gravitational field, one should first mention the successful work of Hayli (1970) concerning the tidal effect on galactic cluster, where the mechanism of disruption of the cluster is analysed in full detail.

Another external effect on galactic clusters, the disruption of clusters through passing clouds of interstellar matter, was investigated by Bouvier and Janin (1970). The disruption time observed in these numerical experiments was found to be roughly two times faster than the theoretical value estimated by Spitzer (1958) corrected by taking into account the close encounters of the clouds with the cluster (Bouvier, 1971).

In the numerical model of Bouvier and Janin, a finite number of idealized clouds of interstellar matter are moving inside a sphere of radius $R$. Whenever one of the clouds hits the surface of the sphere, the direction of its velocity is randomly redistributed so as to send it back into the sphere. This procedure is supposed to reproduce correctly the mean density of the galactic cloud population [a slight mishandling in the procedure as described in the paper of Bouvier and Janin is mentioned by Hénon (1972)]. The star cluster is initially centered at the centre of the sphere. But as soon as a star leaves the central region of the sphere, it undergoes a force towards the centre due to the uniform distribution of clouds.

It would be feasible to compensate for this force by introducing an artificial gravitational field. This field should induce a centrifugal force, whose intensity depends on the mean cloud mass inside a concentric sphere of radius equal to the distance of the star to the centre.

But this field, abruptly cut off at radius $R$, creates a discontinuity at the surface of the sphere of clouds and reduces the credibility of this model.

No really satisfactory model has yet been found. 


\section{Cubic Systems for Representing Large Expanding Clusters}

Numerical experiments on a large uniform expanding gravitational system (Janin and Haggerty, 1974) with regard to an application to cosmological systems (Haggerty and Janin, 1974), have only been possible thanks to two crude assumptions, made in order to limit the number of particles involved in the numerical integration:

- reduction of strongly bound subclusters to single particles at their centres of mass;

- division of the system in a net of subsystems of cubical shape having similar global evolution.

The first assumption is reasonably valid for expanding systems, where the probability of a disintegration of a bound subcluster by a passing particle is small.

The second assumption is made in order to restrict the study of the whole system, if homogeneous, to that of one of the cubical subsystem, having the freedom to associate several subsystems side by side.

Studying only a part of a system gives birth to the problem of taking into account the exterior of the subsystem. An external field is introduced for this purpose. This field is equivalent to having a fictitious uniform negative mass density in the region containing particles. A central attractive field that is linear in the displacement from the centre is added. The spurious effect due to the absence of spherical symmetry is therefore counter-balanced. As the system is expanding, so the additional field is time dependent.

As in the spherical system described in the preceding section, the delicate point is the boundary effects. A systematic study has been made to measure the effect of the cube sides on near-boundary particles.

A general conclusion is that the motion of the particles near the boundary is severely affected by the presence or absence of discrete particles, as distinct from either a uniform fluid or vacuum, on the other side of the boundary. However, the nature of the evolution of small regions is not greatly affected by changes in the mass distribution within other small regions at a great distance.

Among the deviations of parameters observed under the effect of the external field, the most significant encountered is that of the total energy (constant for isolated systems) of the simulated system. In some cases, the magnitude of the energy variation is such that the virial ratio, initially equal to 2 , decreased to 1 when the system expanded by a factor 4 in radius. Later, the energy variation slows down abruptly and can even reverse itself.

It was expected to observe in this representation an energy exchange between the exterior and the interior of the system because of the non-symmetric role played by these two parts: the interior undergoes an evolution while the exterior keeps being a continuous distribution of matter. The initial direction of the energy exchange is shown by the escapers from the interior to the exterior. But the magnitude of the energy stream was a surprise. No completely satisfactory explanation has yet been found. 


\section{References}

Bouvier, P.: 1971, Astron. Astrophys. 14, 341.

Bouvier, P. and Janin, G.: 1970, Astron. Astrophys. 9, 461.

Haggerty, M. J. and Janin, G.: 1974, Astron. Astrophys. 36, 415.

Hayli, A.: 1970, Astron. Astrophys. 7, 17.

Hénon, M.: 1972, Astron. Astrophys. 19, 488.

Janin, G. and Haggerty, M. J.: 1974, J. Comput. Phys. 16, 76.

Spitzer, L.: 1958, Astrophys. J. 127, 17.

\section{DISCUSSION}

Bouvier: In connection to our former investigation about the influence of clouds on star clusters, I wish to point out that we had been led at a disruption time which appeared shorter than the Spitzer disruption time of 1958 , by a factor 3 or 4 . The Spitzer scheme did not take into account either the inner evolution cluster since it was based on the so called 'impulsion approximation' or the closest encounters of the cluster with the clouds, and it was only after having improved the Spitzer deduction that a fair agreement was reached between the disruption times completed numerically and estimated theoretically, in spite of the neglect of the steady-mean field of the clouds in the numerical experiment.

Wielen: It seems to me absolutely necessary to compensate somehow the unrealistic effect of the mean field of the clouds in numerical experiments. As I pointed out to Dr Janin in 1972, I fear that the results obtained by Bouvier and Janin (1970) are severely affected by the unrealistic simulation of the clouds. The unrealistic mean gravitational field towards the center of the cluster, due to the average density of clouds inside a sphere of radius $r$ around the cluster, is equal to the gravitational attraction by the cluster at $r=8 \mathrm{pc}$, and is 30 times as large as that of the cluster for $r \geqslant 26 \mathrm{pc}$. Hence nearly no star could escape from the system. Most of the observed change in the total energy is probably due to a few stars in the outermost regions absorbing much energy from the clouds instead of escaping. In this case, the dissolution time of the cluster as derived from monitoring the total energy of all the stars, is certainly too short. A model in which the mean field of the clouds is just compensated by an appropriate opposite field, should provide more reliable results.

Hohl: Could many of the difficulties associated with the external field be eliminated by using Fouries transform techniques to obtain the field for periodic systems? The field for the central system would still be for the isolated system.

Janin: The use of Fourier transform techniques for estimating the field for periodic gravitational systems is certainly of high interest for our problem, providing a satisfactory handling of the three-dimensional case is available.

Lynden-Bell: In your last experiment, do you start with everything within the cube expanding?

Janin: Yes. We give initial peculiar radial velocities to the particles within the cube which is also expanding at the same rate, more or less like a fluid.

Lynden-Bell: Is it a bound system?

Janin: Yes.

Lynden-Bell: If you have a bound system and you give it a radial pulsation that converts kinetic energy into potential energy the virial ratio goes down. 